\title{
Northern perspectives on medical elective tourism: a qualitative study
}

\author{
Sarah Coke MD, Ayelet Kuper MD DPhil, Lisa Richardson MD MA, Anita Cameron BA
}

Abstract

Background: The Royal College of Physicians and Surgeons of Canada recognizes education to be necessary for doctors to provide culturally safe care. Communities in northern Canada have large populations of Aboriginal people and other marginalized groups. Our goal was to identify the elements of appropriate predeparture curricula for these medical trainees.

Methods: We conducted our study in Kenora, Ontario. With the help of a core collaborative group and the support of the local Aboriginal Health Access Centre, we interviewed a purposive sample of community members about their interactions with trainees from southern Canada. Aboriginal and non-Aboriginal researchers with roots in northern and southern Canada brought perspectives to the inductive analysis.

Results: We conducted 17 semistructured interviews between February and March 2014. Participants felt that southern trainees were inadequately educated in northern politics, society and history. They identified 2 more themes: determinants of health affecting the local Aboriginal population, and provider and patient factors affecting delivery of culturally competent care. Participants also shared ideas on how best to implement this content into curricula.

Interpretation: Providing culturally competent care to northern communities is a complex process requiring education. Using a collaborative method, we were able to delineate the experiences of members of a northern community and identify knowledge gaps of southern trainees travelling there. Our results provide a foundation for the content and structure of formal predeparture curricula to enable such trainees to provide culturally safe care.

$\mathrm{P}$ opulation health studies report that Aboriginal people (a group that includes Canada's First Nations, Inuit and Metis people) continue to have poorer health and shorter life expectancies than the Canadian population as a whole. ${ }^{1,2}$ New research has indicated that Aboriginal people are also less likely to seek medical attention from urban health care services because of feeling "judged, ignored, stereotyped, racialized and minimized." 3 Across Canada, efforts are being made to improve health care delivery and address the health inequities of Aboriginal people. These efforts are meant to create health care environments "free of racism and stereotypes, where Aboriginal people are treated with empathy, dignity and respect." ${ }^{3}$ For instance, urban health centres in numerous provinces have created "Aboriginal patient navigators" to help reconcile relationships between Aboriginal patients and Western health care providers. ${ }^{3}$ In British Columbia, the Provincial Health Services Authority launched an online module called the Indigenous Cultural Competency Training Program, which is the first province-wide mandatory cultural training program for health authorities. The importance of such initiatives is highlighted in a recent report by the Truth and Reconciliation Commission of Canada about the experiences of Aboriginal students in residential schools. ${ }^{4}$ The report specifically calls on health care institutions to train their provid- ers in cultural safety and to teach them about how traumatic residential school experiences have engendered substantial personal and social costs for the survivors.

Since 1972, more than 2000 students from southern Ontario have participated in electives in northwestern Ontario through the Northwestern Ontario Medical Programme. ${ }^{5}$ Trainees continue to travel to Canadian rural communities but may not have prior knowledge about the social and cultural circumstances of the communities. Frequently, communities accommodating trainees have substantial populations of Aboriginal people. In keeping with the World Health Organization's report on social accountability, medical schools are encouraged to focus educational and research initiatives on the specific health needs of the communities they serve. ${ }^{6}$ Physicians, residents and medical students travelling to northern communities,

Competing interests: Lisa Richardson reports salary support from the AMS Phoenix Fellowship for the creation of curricula related to indigenous health during the conduct of the study. No other competing interests were declared.

This article has been peer reviewed.

Correspondence to: Sarah Coke, sarah.coke@mail.utoronto.ca

CMAJ Open 2016. DOI:10.9778/cmajo.20160001 
whether for a short or long period, owe a duty to the community that includes prerequisite education. Similarly, the Indigenous Physicians Association of Canada (IPAC) and the Royal College of Physicians and Surgeons of Canada (RCPSC) have recognized that education is essential to improving care to Canada's Aboriginal people, both in northern communities and in urban environments. In 2009, the IPAC-RCPSC Advisory Committee developed a core training module to help residents and physicians understand the "social, political, linguistic, economic and spiritual realm" of First Nation, Inuit and Metis patients. ${ }^{7}$ Despite these initiatives, no nationwide mandate currently exists requiring health care providers or medical trainees to complete education on cultural competency.

The objective of the study was to learn from the inhabitants of a rural Canadian community what medical students and residents need to know, learn and experience to be adequately educated to travel there as part of their medical training or subsequent practice.

\section{Methods}

\section{Setting}

Our research was conducted in Kenora, a town of 15348 people in northwestern Ontario, near the Manitoba border. Kenora has a large Aboriginal population, with 2365 (15.4\%) inhabitants reporting "Aboriginal identity" (defined as either Metis, Inuit, North American Indian Status or Non-Status First Nation), making it the largest concentration of Aboriginal inhabitants of all Ontario towns. ${ }^{8}$ Kenora is surrounded by 13 Aboriginal reservations, including Rat Portage, Shoal Lake and Ochiichagwe'Babigo'Ining Ojibway Nation. Medical students and residents have been travelling to Kenora through the Northwestern Ontario Medical Programme since 1972. This program was a collaborative effort between the Thunder Bay and northwestern Ontario medical societies and McMaster University to recruit and maintain doctors in northwestern rural and remote communities by organizing core or elective rotations. ${ }^{9}$ Participants of this program were not necessarily provided with prerequisite training on the locations they would be travelling to. The long-standing history of trainees coming from southern Ontario and the substantial Aboriginal population made Kenora an ideal location for our research.

\section{Study design and sampling}

Historically, research conducted within Aboriginal communities often did not directly involve the input of Aboriginal people, or reflect their values or beliefs. ${ }^{10}$ Results from projects conducted in these areas, in turn, may not have benefited the people, causing Aboriginal people to view research within their communities with apprehension. ${ }^{11,12}$ To ensure our research was collaborative with the Kenora community, we contacted a local Aboriginal Health Access Centre (Waasegiizhig Nanaandawe'iyewigamig [WNHAC]) before initiating our research project. With the help of key stakeholders at WNHAC, a preliminary research agreement was drafted (Appendix 1, available at www.cmajopen.ca/content/4/2/E277/ suppl/DC1). The document specified commitments regarding "collective community participation and decision making, sharing of benefits and review."13 These concepts are in keeping with the primary principles of research with Aboriginal communities, including mutual responsibilities in project design, data collection and management, as well as ownership, access and possession of data. Ethics approval was obtained from the University of Toronto Research Ethics Boards.

With the help of WNHAC, a preliminary purposive sample of interviewees was created for initial interviews. Eligible participants included, but were not limited to, health care workers and community members who had previously interacted with medical students or residents from southern Ontario medical schools, including the University of Toronto. Potential interviewees were approached via email or in person. Interviews were set up to allow preliminary analysis of results, to allow iterative adjustment to the interview script and study sample. Subsequent interviewees were chosen both through purposive sampling and through snowball sampling (defined as "sampling participants found by asking current participants in a study to recommend others whose experiences would be relevant to the study"). ${ }^{14}$

\section{Data collection and analysis}

Semistructured interviews were conducted by the primary investigator (S.C.). An interview guide was created in collaboration with the primary research team and a representative from WNHAC (Appendix 2, available at www.cmajopen.ca/ content/4/2/E277/suppl/DC1). The interview guide was not piloted. Interviews were conducted in the Lake of the Woods District Hospital, offices of participants or on reserves surrounding Kenora. Interviews were recorded and anonymized onto a digital recording device, then transcribed verbatim by the primary investigator. Interview scripts were reviewed by 2 members of the internal research team (A.K., L.R.). There were no repeat interviews. Constant concurrent comparative analysis allowed theoretic coding of the data within a critical constructivist framework based on decolonizing indigenous research methods. ${ }^{15}$ Saturation of the data was achieved when the conduction of further interviews would not add additional insight to the research question. Themes were refined through discussion among all of the authors who represented indigenous and nonindigenous critical perspectives as well as life experiences in both northern and southern Ontario. The numerous discussions allowed the primary investigator to reflect on and rectify unintended biases or assumptions resulting from personal characteristics or goals for the research project. Purposive sampling was used to address gaps identified in the initial data. The results were reviewed with representatives from WNHAC before submission, as outlined in the Possession section of the research agreement (Appendix 1).

\section{Results}

In total, 17 semistructured interviews were conducted between February and March 2014 to achieve data saturation. None of the participants dropped out or withdrew consent. The mean interview length was 40 (range 8-93) minutes. 
Interviewees consisted of community members and health care employees who self-identified as either Aboriginal or non-Aboriginal. Of the 17 interviewees, 13 were female, and 16 were health care employees.

\section{Southern trainees in northern Ontario}

Of the 17 participants, 13 had previous experience with medical students or residents from either southern Ontario medical schools or the Northern Ontario School of Medicine (NOSM).

Some interviewees commented specifically on negative interactions between the trainees from southern Ontario and members of the Kenora community, all during interactions with the Aboriginal population. Some southern trainees were found to lack a basic understanding of the geographical barriers for Aboriginals to access health care, resulting in poor adherence to treatment plans. Interviewees with direct interactions with trainees from southern Ontario also commented on the trainees' lack of knowledge about important historical events (Table 1).

Many of the health care employees interviewed commented on the difference between trainees from NOSM and southern Ontario. The Northern Ontario School of Medicine was established in 2005 with a mandate to train doctors to serve northern Ontario's urban and remote communities. It is the only Canadian medical school to have developed a core curriculum in Aboriginal health that includes a 4-week Aboriginal cultural immersion placement in year $1 .{ }^{16}$ The interviewees observed that NOSM students had superior baseline knowledge on the historical, political and geographical issues affecting rural communities (Table 2).

\section{Determinants of health affecting the Aboriginal community}

\section{Access to health care}

There was a unanimous consensus that poor access to health care is an important determinant of health among Aboriginal people, especially those residing on reserves outside the city of Kenora. Although some communities had clinics or health centres, many interviewees felt that they were understaffed and/or inadequately equipped to perform simple services such as radiography, blood tests or electrocardiography commonly performed in hospital. Without access to skilled staff or equipment, patients on reserves must find other ways to obtain care, often delaying diagnosis and/or treatment.

The importance of having clinics located on the reserve becomes evident when you consider that some of these communities are hundreds of kilometres from the nearest hospital. During the winter months, many of the roads are inaccessible, and community members may not have access to a vehicle (Table 3). Some of the interviewees commented on an overall improvement in access to health care with the development of roads, the helicopter service operating out of the hospital and physicians travelling to reserves (Table 4).

\section{Residential school influence}

Each of the 17 interviewees commented on the pervasive effect that residential schools continue to have on the local Aboriginal community (Table 5).

\section{Provider- and patient-specific factors affecting delivery of care}

\section{Provider-specific characteristics}

Every interviewee offered perspectives on the characteristics of health care employees that facilitate culturally safe care. Being respectful, empathetic and a good listener were consistently emphasized. In addition to being a good listener, interviewees emphasized good communication skills as essential to facilitating meaningful relationships with patients. Interviewees described being direct and using simple language as effective strategies for communication (Table 6).

\section{Patient-specific factors}

Interviewees commented that some Aboriginal people mistrust the Western health care system or prefer to use traditional medicines. This mistrust is an obvious obstacle in developing comprehensive patient care plans and in creating meaningful patient-provider relationships. The interviewees who were health care employees for the most part were accepting of traditional medicines and their unique role in the overall health of the Aboriginal community. However, they suggested using traditional medications with caution in combination with other pharmaceuticals. Finally, some interviewees explored fundamental cultural differences that may strain relationships between health care provider and patient (Table 7).

Table 1: Participant quotations about students from southern Ontario training in northern Ontario

"I just remember one in particular because we were sharing some of the stuff about residential schools and roles of the elders, stuff like that. He was Canadian, and had no idea, had never heard of this 60 s scoop or anything like that. So that was really shocking to me." (Interview 10)

"Almost always there was very little knowledge in the group. And that's one of the reasons we had the content that we did. Because we realized that you don't get this in your health care training program." (Interview 10)

"The only time they've seen a reserve is Six Nations. So they see these very high end, organized, beautiful places and that's their experience of what a First Nations reserve is like. ... So they don't understand what reserves in the rest of Canada are like. They don't have a good appreciation of residential schools or the Third World living conditions that exist right within our community." (Interview 13)

"I've had a couple individuals from Toronto come down ... I think they were frustrated. ... Without taking into account their patients can't afford to go to Kenora, or they can't afford time off work or the day that it takes with the medical van. That sort of judgment came across and it wasn't appreciated by the population." (Interview 15) 


\section{OPEN}

Research

Table 2: Participant quotations about students from the Northern Ontario School of Medicine and comparison with students from southern Ontario

"You know, since NOSM University has been around, they are way more prepared than the other universities. I think they have a community component to their curriculum." (Interview 1)

"I do know just through a friend that did the Northern Ontario School of Medicine that they receive a lot of education on the First Nation culture. And she did feel prepared for those conversations." (Interview 4)

"Are the northern students that much more aware? I would say they are, but they still have a lot to learn ... in their first 3 months they are out already on their first northern placement. It's not necessarily a clinical one, it's an immersion in the culture and they are sent to different First Nations communities for 4 weeks. So their awareness and knowledge really develops very early in the program. As far as living conditions, social economic conditions, health challenges." (Interview 10)

"There's 5 NOSM students right now, some of them are from the North. ... I think they have a better appreciation and understanding of the demographic in the region than someone coming from Toronto who have never maybe been on a reserve. ...I think a lot of the students we have right now from NOSM have a pretty good appreciation of the social deprivation that exists in some of these far north communities. ... So I think there is a difference, I think for some of our students that we had from the Toronto area, aren't familiar with the North." (Interview 11)

Note: $\mathrm{NOSM}=$ Northern Ontario School of Medicine.

Table 3: Participant quotations about access to health care

"Yes, there are clinics in the community. Some of them have everything and some of them have nothing. So we have to bring our own stuff like pap lights and blood pressure cuffs." (Interview 1)

"No, we don't have ECGs, there's no testing done here. We're more of a health centre than a medical centre. The only professionals that are here are the nurses and the nurse practitioner." (Interview 5)

"I'm waiting for an answer to see when we're going to get our new health care facility. This one is very small. We're overcrowded. We don't have a lot of places to put storage. We don't have anywhere to put medical files." (Interview 5)

"Some communities have nursing stations that have home care services or have medical drivers that can get people to town. But ... if a student doesn't realize that someone might not be able to get to town because it's over an hour away and it's $\$ 100 \ldots$ that's going to have an impact on [the patient's] health." (Interview 11)

"It's $110 \mathrm{~km}$ north of Kenora, so it takes just over an hour to drive. Sometimes the road's out, which always makes it exciting. ... It's a regular road but several areas along the road they have washouts because of water, and culverts are not maintained.... And often they have to close it for repairs, so you can travel through it. They only open it up to let people through for 1 hour a day, so it really limits the travel to the community. (Interview 15)

Note: $E C G$ = electrocardiogram

Table 4: Participant quotations about improved access to health care

"Probably the access to the hospital is easier now than it was 40 years ago because of roads and because of the helicopter. The helicopter brings a lot of people from the reserves." (Interview 3)

"A lot of the family doctors have adopted a First Nations community. So they are receiving care at that level too." (Interview 4)

"The outreach of physicians going to the communities has had a world of change." (Interview 16)

Table 5: Participant quotations about the influence of residential schools

"There are a lot of negative impacts that happened from the residential school, and that goes into each generation." (Interview 7)

"The history of colonization and residential schools, how that has affected our population here ... people don't understand, they don't make the connection that your ER patient today, how it affected their family and their mothers, their grandparents or themselves." (Interview 8)

"Generations of disruption, that nobody knows how to raise kids, survive as a family, survive without intoxicants which help you forget about all the other stuff that you don't want to remember." (Interview 13)

"So, we are trying to deal with the residential school and those impacts. We try to have sharing circles and I think we need to work on that awareness. The healing has to happen from their own hearts." (Interview 14)

"I expected to see the legacy of the residential schools and that would all be starting to fade and we'd be seeing a light, but it's no different now than it was 20 years ago. In fact, in some cases it's worse." (Interview 16)

$\mathrm{ER}=$ emergency room. 


\section{New curriculum for education of trainees from southern Ontario}

All of the 17 interviewees felt that a predeparture curriculum would be beneficial for trainees coming from southern Ontario (Table 8). Interviewees also identified the need to instruct trainees on some of the nuances surrounding effective communication with the Aboriginal population (Table 9).

In addition to a predeparture curriculum, the interviewees suggested an immersive experience to teach trainees about the complexities of northern Ontario. This experience could include spending time in an Aboriginal community, having storytelling sessions with native elders or participating in traditional ceremonies (Table 10). Finally, some of the interviewees discussed the concept of mentorship (having a preceptor to help with the educational and practical aspects of a placement) as beneficial for students and residents coming to northern Ontario for the first time (Table 11).

\section{Interpretation}

Using a collaborative method, we were able to gain the perspective of a northern Ontario community about what elements should be incorporated into a predeparture curriculum for southern Ontario trainees wishing to travel there on elective. The interviewees who had worked previously with trainees from southern Ontario felt that the trainees were inadequately educated about northern politics, society and history. All interviewees felt that a predeparture curriculum would be beneficial to mitigate deficiencies in knowledge. They agreed unanimously about the content, that it should include information on the political, historical and social determinants of health affecting the Aboriginal populations in northern Ontario. They also felt education on historical events like the Indian Act is important in understanding the current health structure and how services in care delivery are organized for the Aboriginal population. Incorporating didactic sessions with an integrative immersive approach while in northern Ontario was felt to be a more effective way for students to understand the area and to collaborate more successfully with the Aboriginal community. The importance of good mentorship during the placement and education to improve communication were also identified. As a result, the trainees will feel more prepared, deliver appropriate, culturally competent care and have an overall better educational experience.

A major determinant of health affecting the Aboriginal community in Kenora includes unreliable access to health care for remote reserves, which has previously been described in the literature by the National Collaborating Centre for Aboriginal Health. ${ }^{17}$ Interviewees discussed efforts made to improve health care access, including doctors and nurses travelling to reserves and the helicopter service available for emergency transportation of patients to the hospital. Another determinant discussed by the interviewees included the pervasive effect of local residential schools. Kenora was home to 3 residential schools, the last one closing in $1969 .{ }^{18}$ Most of the Aboriginal community would have had family members who attended these schools. To this day, the

Table 6: Participant quotations about provider-specific characteristics influencing care delivery

"I think if you're dealing with somebody who has some degree of empathy, and patience and a little bit of understanding, I mean, you don't need anything more than that." (Interview 3)

"Being respectful and kind. I think that of many different cultures, so if you're being respectful to an individual and asking them, "How can I help you?", I think that can be perceived too as appropriate cultural care." (Interview 4)

"When I have students with me I really remind them to have good listening skills. This sometimes means a lot of time and not talking. I do believe that you can then deliver quicker health care in the longer term because you can understand the patient and you're probably going to get better compliance." (Interview 10)

"White health care people have not walked in the same types of shoes. We are so quick to talk and to give information and instructions and recommendations and with the First Nations population, you have to be a good listener and give time for someone to tell their story." (Interview 10)

"Learn how to listen to the stories that the elders have to share. Sometimes the stories are pretty long but being able to really listen to them and take the little seeds that are pearls of wisdom, and if you learn how to do that you're going to benefit an awful lot. And you're going to find the time you're in that First Nation community a lot better, a lot easier." (Interview 17)

Table 7: Participant quotations about patient-specific factors influencing care delivery

"We do have part of our community that's at the far end and they are very untrusting. The grandfather got a flu shot and the next day he died. So they really thought that it was the flu shot that killed him. And I think to this day it's really hard to break that barrier." (Interview 5)

"And l'm fine with people using native medicine; they need to be careful about interactions because there are some very powerful roots and herbs." (Interview 13)

"I think from a mental health perspective, the sweats that they do and the sage smoking are very therapeutic.... It is a negotiation with those patients to come to some sort of common ground to say, I'm okay with you using your traditional medicine but I think it's in your best health interest to also consider using Western medicine ... to optimize the treatment for that condition." (Interview 15)

"It's understanding the very different attitude just towards parenting, towards social intervention, the concept of noninterference, which is totally at odds with our jobs. ... Western medicine is based on a concept of direct interference and the First Nations approach is noninterference, and it applies to parenting and it applies to health care and applies to justice." (Interview 16) 


\section{OPEN}

Research

historical implications of these schools continue to resonate within the community. The concept of historical trauma in one generation influencing subsequent generations has been described in the literature. ${ }^{19}$ Survivors of the Canadian residential school system experienced trauma in the form of physical, mental and sexual abuse, as well as separation from their families. Not surprisingly, evidence has found that children of residential school attendees have higher rates of substance abuse and depressive symptoms. ${ }^{20,21}$ Therefore, the history of Canadian residential schools should be part of a curriculum describing the current health issues of Aboriginal people in northern Ontario.

Another theme uncovered from the interview scripts included provider- and patient-specific factors influencing culturally safe care to the Aboriginal population. The interviewees emphasized respect, patience and understanding as important provider characteristics. The themes brought up by the interviewees are in keeping with IPAC and the RCPSC core competencies published in 2009. ${ }^{7}$ The Communicator section suggests that "reciprocity, equality, trust, respect, honesty and empathy" are required during interactions with the Aboriginal community, as with all patients. ${ }^{7}$ Additionally, some interviewees discussed the principle of "noninterference" as a fundamental backbone of Aboriginal culture and its impact on patient-provider relationships. Noninterference is a concept described by Rupert Ross in his book Dancing with a Ghost, which encapsulates the importance of allowing individ-

\section{Table 8: Participant quotations about the necessity for and content of a predeparture curriculum}

"It would be good for them to be prepared so that when they come they are aware of the stressors in the community.... And what did it stem from." (Interview 4)

"You could probably do an introductory type session; go over the pertinent social problems. Maybe some of the social dynamic issues about negotiating with patients and that you are not going to get everything on your optimal care list. And what's available, the cost of transportation, what's involved time-wise. Because in Toronto everything's there. And the expectation when they go elsewhere is that it should be there." (Interview 15)

"I think it's a really good idea to go over that before you come because then when you have that first patient in your office who has multiple comorbidities, you have an understanding of where they're coming from. ... So certainly a historical perspective would make it a little easier." (Interview 16)

"Knowing a little bit about the geography, some of the history of the community, of the First Nations people. Certainly ... an understanding of the Indian Act, the residential schools and the impact that it had over the generations." (Interview 17)

Table 9: Participant quotations about education on communication

"An appreciation for how to communicate, knowing that not looking people in the eye is not rude, if people don't look at you it's not that they're ignoring you." (Interview 13)

"Some cultural interactions, like how to interact with the First Nations people ... they don't make eye contact, they often make a number of gestures. [Making] an effort to make or to learn a few words in their local language goes a long way to develop more rapport."

(Interview 15)

Table 10: Participant quotations about an immersive experience for trainees

"I think if they attend a sweat lodge ceremony and learn the rationale behind it ... You will gain a better perception of what the cultures and beliefs are." (Interview 6)

"I think every doctor that comes out should have some training in a First Nations community." (Interview 7)

"We have had some storytelling from elders ... that is the most profound understanding that you can get." (Interview 8)

"I think that maybe they can be given some resources or something in advance to help orient themselves. But I think it's really successfully done as an immersion approach. When you're here, you can learn, integrate and apply all at the same time, simultaneously." (Interview 10)

"There's nothing that will overtake hands-on experiential learning. If everybody had a half day or a day of immersive learning ... it would add a lot to their teachings." (Interview 13)

"I think spending time on a First Nations community is probably the best way to really understand and appreciate the health care challenges." (Interview 16)

\section{Table 11: Participant quotations about the importance of mentorship}

"Say, if you're going to work for someone like WNHAC, you know the people there are really awesome. They don't need to, but they'll bring you with them and they will introduce you, kind of ease you into it." (Interview 5)

"When people come and go for short term, it's the best when they go with the physicians because the physicians are really community involved ... we are their gateway to the community for the students and residents." (Interview 13)

Note: WNHAC = Waasegiizhig Nanaandawe'iyewigamig Health Access Centre. 
uals to make their own mistakes and not to interfere with the behaviours of others. ${ }^{22}$ This principle conflicts with the patriarchal aspects of Western health care, in which doctors are supposed to interject and guide patients toward good health care decisions. Understanding cultural differences such as this can help doctors and health care employees understand why resistance may exist, and how to collaborate more effectively.

\section{Strengths and limitations}

The research was limited to one of many towns in the vast expanse of northern Ontario. Each town or city in northern Ontario undeniably encompasses its own set of values and nuances, as does every Aboriginal community. The opinion of what should be incorporated within a curriculum was based on the perspectives of health care employees and community members, but not trainees. Strengths of the research included the use of a collaborative method, with community representatives as active research team members in project design and execution. A research agreement was created between the primary researchers and a local Aboriginal Health Access Centre (WNHAC) to ensure collaboration regarding ownership, access and possession of all data obtained during the research. Our methods (including the use of a research agreement) can be used as a template for future research conducted in Aboriginal or other communities in which a community-based research initiative is needed. This study also provides an example for non-Aboriginal researchers about how to partner with Aboriginal communities in a meaningful way to bring about transformative change. Such partnerships and allegiances are a critical step toward reconciliation and in moving forward with reciprocal respect to improve the care of Aboriginal people in Canada.

\section{Conclusion}

Using a collaborative method, we were able to gain the perspectives of a northern Ontario community about past experiences with southern Ontario trainees and about how to best implement a predeparture curriculum. Based on our research findings, we suggest that every trainee who travels to northern Ontario for a clinical or research elective should be required by their university to receive predeparture training in the areas of cultural safety and indigenous health. Formal curricula offered by the individual university would ideally be the product of a collaborative effort between medical education experts and representatives from the Aboriginal community. A general predeparture training session or curricula must acknowledge the diversity of northern communities and consider the specific needs, strengths and resources within the destination community. On arrival in a community, each trainee should meet with and learn from local partners to adapt their general teaching about indigenous health to the specific context of their host community. These local opportunities for learning can both strengthen existing partnerships between medical schools and community sites, and underscore the richness of indigenous knowledge and the critical role that indigenous people play in the education of health professions. Educational efforts such as these will ensure culturally safe care is being provided to Canada's Aboriginal population and, as a result, the health inequities in this population may start to improve.

\section{References}

1. MacMillan HL, MacMillan AB, Offord DR, et al. Aboriginal health. CMAf 1996;155:1569-78.

2. Adelson $\mathrm{N}$. The embodiment of inequity: health disparities in aboriginal Canada. Can 7 Public Health 2005;96:S45-61.

3. Empathy, dignity and respect: creating cultural safety for Aboriginal people in urban bealth care. Toronto: Health Council of Canada; 2012.

4. Honouring the truth, reconciling for the future: summary of the Final Report of the Truth and Reconciliation Commission of Canada. Winnipeg: Truth and Reconciliation Commission of Canada; 2015. Available: www.trc.ca/websites/trcinstitution/File/2015/ Findings/Exec_Summary_2015_05_31_web_o.pdf (accessed 2015 June 2).

5. McCready W, Jamieson J, Tran M, et al. The first 25 years of the Northwestern Ontario Medical Programme. Can 7 Rural Med 2004;9:94-100.

6. Boelen C, Heck JE. Defining and measuring the social accountability of medical schools. Geneva: World Health Organization; 1995.

7. Promoting culturally safe care for First Nations, Inuit, and Metis patients: a core curriculum for residents and physicians. Ottawa: IPAC-RCPSC Core Curriculum Development Working Group; 2009.

8. Kenora O. (Code3560010) (table). Aboriginal population profile. 2006 Census. Statistics Canada cat. no. 92-594-XWE. Ottawa: Statistics Canada; 2008 Jan. 15. Available: www12.statcan.ca/census-recensement/2006/dp-pd/prof/92 $-594 /$ details/page.cfm? Lang $=\mathrm{E} \&$ Geo1=CSD\&Code1 $=3560010 \& \mathrm{Geo}=\mathrm{PR} \&$ Code2 $=35 \&$ Data $=$ Count $\&$ SearchText=Kenora\&SearchType=Begins $\&$ Search $\mathrm{PR}=01 \& B 1=A l l \&$ GeoLevel $=P R \&$ GeoCode $=3560010$ (accessed 2012 Dec. 1$)$.

9. McCready W, Jamieson J, Tran M, et al. The first 25 years of the Northwestern Ontario Medical Programme. Can f Rural Med 2004;9:94-100.

10. Quigley D. Perspective: a review of improved ethical practices in environmental and public health research: case and examples from native communities. Health Educ Behav 2006;33:130-47.

11. Davis SM, Reid R. Practicing participatory research in American Indian communities. Am 7 Clin Nutr 1999;69(Suppl):755S-9S.

12. Maddocks I. Ethics in aboriginal research: A model for minorities or for all? Med f Aust 1992;157:553-5.

13. Research involving the First Nation, Inuit and Metis Peoples of Canada. Ottawa: Panel on Research Ethics; 2013. Available: www.pre.ethics.gc.ca/eng/policy -politique/initiatives/tcps2-eptc2/chapter9-chapitre9 (accessed 2015 June 2).

14. Kuper A, Lingard L, Levinson W. Critically appraising qualitative research. BM7 2008;337:a1035.

15. Smith LT. Decolonizing methodologies: research and indigenous people. London (UK): Zed Books; 1999

16. Jacklin K, Strasser R, Peltier I, et al. From the community to the classroom: the Aboriginal health curriculum at the Northern Ontario School of Medicine. Can f Rural Med 2014;19:143-50.

17. Access to bealth services as a social determinant of First Nations, Inuit and Métis health. Prince George (BC): National Collaborating Centre for Aboriginal Health; 2009. Available: www.nccah-ccnsa.ca/docs/fact\%20sheets/social\%20determinates/Access $\% 20$ to\%20Health\%20Services_Eng\%202010.pdf (accessed 2015 June 2).

18. List of residential schools. Waterloo (ON): Indian Residential Schools Settlement - Official Court Website. Available: www.residentialschoolsettlement. ca/schools.html\#Ontario (accessed 2015 Mar. 3).

19. Evans-Campbell T. Historical trauma in American Indian/Native Alaska communities: a multilevel framework for exploring impacts on individuals, families, and communities. 7 Interpers Violence 2008;23:316-38.

20. Bombay A. The intergenerational effects of Indian Residential Schools: implications for the concept of historical trauma. Transcult Psycbiatry 2014;51:320-38.

21. Bombay A, Matheson K, Anisman H. The impact of stressors on second generation Indian residential school survivors. Transcult Psycbiatry 2011;48:367-91.

22. Ross R. Dancing with a ghost: exploring Indian reality. Toronto: Penguin; 2006.

Affiliations: Department of Medicine (Coke, Richardson), Faculty of Medicine (Kuper), University of Toronto, Toronto, Ont.; Waasegiizhig Nanaandawe'iyewigamig Health Access Centre (Cameron), Kenora, Ont.

Contributors: Sarah Coke was the main author and contributed to the project design, data collection and interpretation of data. Ayelet Kuper contributed to the project design and data analysis. Lisa Richardson contributed to the project design, data analysis and interpretation of data. Anita Cameron contributed to the project design, participant recruitment and data analysis. Sarah Coke wrote the manuscript, which all of the authors revised. All of the authors gave final approval of the version to be published and agreed to act as guarantors of the work.

Supplemental information: For reviewer comments and the original submission of this manuscript, please see www.cmajopen.ca/content/4/2/ E277/suppl/DC1. 